\title{
Synaptoimmunology - roles in health and disease
}

\author{
Robert Nisticò ${ }^{1,2^{*}}$ (D) Eric Salter ${ }^{3}$, Celine Nicolas ${ }^{4}$, Marco Feligioni ${ }^{2}$, Dalila Mango ${ }^{2}$, Zuner A. Bortolotto ${ }^{4}$, \\ Pierre Gressens ${ }^{5,6}$, Graham L. Collingridge ${ }^{3,4}$ and Stephane Peineau ${ }^{4,5,7^{*}}$
}

\begin{abstract}
Mounting evidence suggests that the nervous and immune systems are intricately linked. Many proteins first identified in the immune system have since been detected at synapses, playing different roles in normal and pathological situations. In addition, novel immunological functions are emerging for proteins typically expressed at synapses. Under normal conditions, release of inflammatory mediators generally represents an adaptive and regulated response of the brain to immune signals. On the other hand, when immune challenge becomes prolonged and/or uncontrolled, the consequent inflammatory response leads to maladaptive synaptic plasticity and brain disorders. In this review, we will first provide a summary of the cell signaling pathways in neurons and immune cells. We will then examine how immunological mechanisms might influence synaptic function, and in particular synaptic plasticity, in the healthy and pathological CNS. A better understanding of neuro-immune system interactions in brain circuitries relevant to neuropsychiatric and neurological disorders should provide specific biomarkers to measure the status of the neuroimmunological response and help design novel neuroimmune-targeted therapeutics.
\end{abstract}

Keywords: Nervous system, Immune system, Synaptic plasticity, Neuroinflammation, Microglia

\section{Introduction}

Following a pathogenic insult to the brain, most central nervous system (CNS) cells, as well as some peripheral immune cells, participate to the genesis of a central inflammation known as neuroinflammation. This process consists of complex biochemical cascades that serve as a protective mechanism to eliminate the initial cause of cell injury and promote recovery. For many years it was thought that the immune system within the CNS served exclusively a reactive role following insults to the brain. However, recent evidence suggests that the brain and immune system are intimately linked and engage in significant cross-talk under physiological, not just pathological, conditions to preserve homeostasis. Indeed, several proteins first detected in the immune system have been found also in the healthy uninfected nervous system, where they are having pleiotropic functions. Conversely, proteins first described in the nervous system have since been associated with immunological functions [1]. These factors influence

\footnotetext{
*Correspondence: robert.nistico@uniroma1.it; stephane.peineau@inserm.fr 1 Department of Biology, University of Rome Tor Vergata, 00133 Rome, Italy ${ }^{4}$ Centre for Synaptic Plasticity, School of Physiology, Pharmacology \& Neuroscience, University of Bristol, Bristol, UK

Full list of author information is available at the end of the article
}

numerous physiological functions including neurite outgrowth, neurogenesis, neuronal survival, synaptic pruning, synaptic transmission and synaptic plasticity [2]. We have termed the interplay between immune modulators and synaptic function, synaptoimmunology.

In this review we first provide a summary of the mechanisms of synaptic transmission/plasticity and immune cell signaling pathways. Then, we discuss how these pathways converge and thus play a role in numerous physiological functions of synapses, with an emphasis on synaptic plasticity. Finally, we describe how synaptoimmunology is involved in a variety of different brain disorders.

\section{Synaptic communication}

Synapses are the main points of rapid communication between neurons (and in some cases between neurons and astrocytes or microglia), through the use of chemical neurotransmitters. This communication is subject to alteration, a phenomenon known as synaptic plasticity: synaptic strength can be enhanced (potentiation, P) or reduced (depression, D). The duration of the alteration could be transient (on the order of $\mathrm{s}$ and $\mathrm{min}$ ) or more stable (h to years) and is defined as short term (ST) or long term (LT) respectively. Both parameters define the 
type of plasticity occurring at the synapses: LTP, LTD, STP, STD. Mechanistically, synaptic plasticity modulates either the function of membrane proteins (gain or loss) or their availability (endocytosis, exocytosis, degradation, synthesis). All these events are under the control of multiple intracellular signaling pathways [3-5].

Neurons are also able to communicate through volume communication, mainly via diffusing peptide molecules (e.g., neuropeptides, cytokines, growth factors). This communication can arise between neurons but also between the different cell types of the CNS (neurons, astrocyte, microglia, oligodendrocytes, endothelial cells, immune cell, etc.). These peptide molecules are more slowly degraded or captured compared to the smaller sized transmitters and can thus diffuse far from their release site. Once they reach their target (GPCR, kinase receptor, etc.), intracellular signaling pathways are activated. On neurons, these receptors can be located at, or in the vicinity of, synapses, where they can directly modulate synaptic functions [6-8].

\section{Cell signaling at synapses}

The activation of intracellular signaling pathways at synapses, as a response to synaptic events or diffusing molecules, can lead to the modification of the local synaptic strength and also a more generalized alteration in neuronal function that often involves changes in gene expression. Consequences of this signaling can therefore be restricted to a local action mode within, or in the vicinity of, the stimulated synapse, or can involve interactions between the soma and synapses.

Synaptic strength is modified by synaptic plasticity events. During LTP, some kinases cascades, such as CaMKII, have a strict local action as they are involved in synaptic cytoskeleton remodeling, AMPAR trafficking and/or local protein synthesis. The PKA cascade, however, can both affect glutamatergic receptor properties locally as well as regulate somatic transcription and translation [9-11]. Considerable cross-talk exists between these different cascades. A similar scheme also exists for LTD: PP1 or PLC cascades modify AMPAR trafficking and internalization, whereas JAK/STAT, PI3K and eEF2K have both local and somatic roles (Fig. 1) [3, 12].

Whilst most forms of synaptic plasticity are induced by activation of glutamatergic receptors, synapses express numerous other receptors including neuropeptide receptors, cytokine receptors, growth factor receptors, which represent potentially hundreds of receptors able to detect circulating molecules. Interestingly, many of these receptors engage the same signaling pathways as those involved in synaptic plasticity. This potentially enables many ways in which neuropeptides, and other neuromodulators, can affect synaptic plasticity and other synaptic functions (Fig. 2).

\section{Immune system signaling}

The immune system acts to defend against and restore homeostasis following the invasion of foreign pathogens

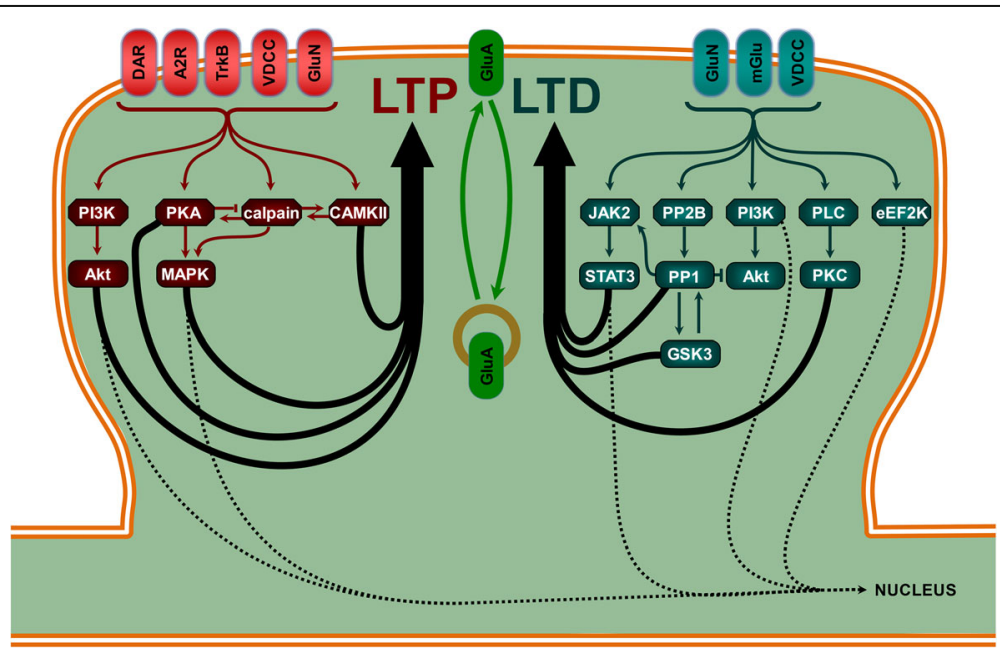

Fig. 1 Some of the main signaling pathways in LTP and LTD. LTP involves (at different synapses) several type of receptors which include NMDA receptor (GluN), voltage dependent calcium channel (VDCC), neurotrophin receptor (trkB), adenosine 2 receptor (A2R) or dopamine receptor (DAR). These receptors activate intracellular signaling pathways with local and/or somatic effects, such as phosphoinositide-3 kinase (PI3K)/Akt, protein kinase A (PKA)/ mitogen activated protein kinases (MAPK), calpain/ striatal enriched protein tyrosine phosphatase (STEP) and calcium calmodulin kinase II (CaMKII) pathways. LTD can be triggered by the activation of, for example, GluN, VDCC and metabotropic glutamate receptors (mGlu), depending of the form of LTD. Calcineurin (PP2B)/protein phosphatase 1 (PP1) associated to Janus kinase 2 (JAK2)/ signal transducers and activators of transcription 3 (STAT3), PI3K/Akt and glycogen synthase kinase 3 (GSK3) are mainly required for GluN dependent LTD whereas mGlu dependent LTD activates mainly phospholipase C (PLC)/Protein Kinase C (PKC) and eukaryotic elongation factor 2 kinase (eEF2K) signaling pathways. Sequence of activation of these pathways and inter-regulation between them are two key features to obtain synaptic plasticity events 


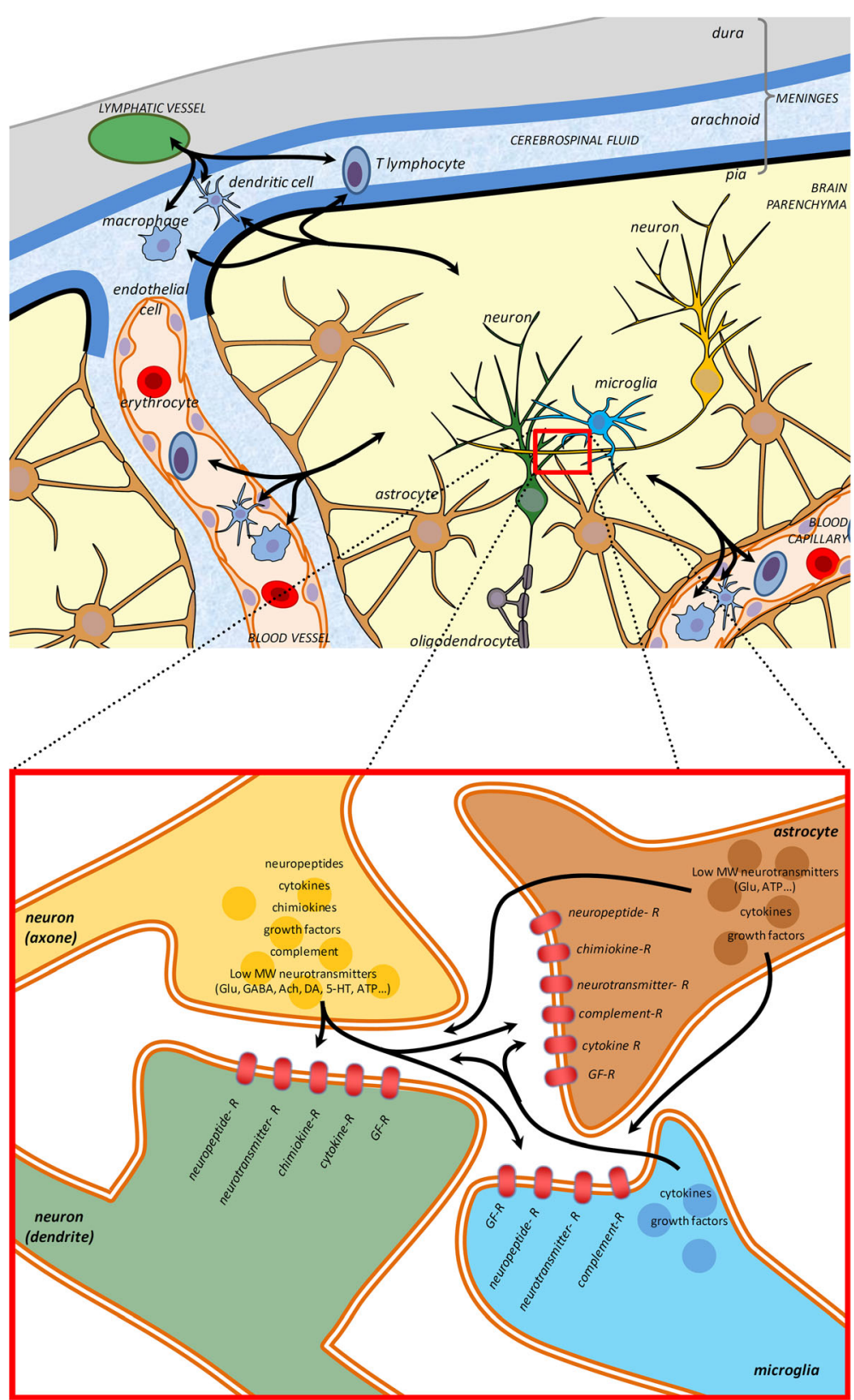

Figu. 2 A schematic of a synapse showing pre, post elements, astrocytes and microglia. Brain and immune cells undergo a dynamic dialog. Peripheral immune cells, such as T-lymphocytes, macrophages and dendritic cells, coming from the cerebrospinal fluid or carried by blood vessels penetrate the brain through the blood brain barrier. They either have a surveilling activity or are attracted by the chemokines released by injured tissues. Microglia, the brain resident immune cells, perform a constant surveilling activity and are in particular attracted by synapse activity, locus of an intense interplay between neurons and glial cells. Many neurotransmitters circulate between these cell types resulting in the modulation of the synaptic functions. Increasing evidence suggest that molecules and signaling pathways first discovered for the immune system takes an important place in the physiological functioning of the synapse. Growth factor receptor (GF-R); Glutamate (Glu); Gamma Amino Butyric Acid (GABA); Acetylcholine (Ach); Dopamine (DA), Serotonin (5-HT), Adenosine tri-phosphate (ATP). (top scheme, cerebral structure inspired from [37])

and tissue damage. This is achieved by the two arms of the immune system, the innate and adaptive systems, the former being a more rapid, nonspecific response while the latter is slower and specific to a particular antigen. Pattern Recognition Receptors (PRR) on the surface immune cells detect damage associated molecular patterns (DAMPs, like heat shock proteins, ATP, cell fragments or mRNAs) released following tissue damage as well as pathogen associated molecular patterns (PAMPs, such as lipopolysaccharide) found on the surface of microbes. Activation of Toll-like receptors (TLRs), in cooperation with other PRRs, leads to the 
expression and release of cytokines and other inflammatory molecules (TNF, IL-1 $\beta$, IL-6, NO, etc.), via activation mainly of NF B, MAPK and caspase-1 [13, 14]. The factors released attract other immune cells and activate a variety of specific receptors. Depending on the receptor, different signaling pathways can be activated, all leading to the modulation of genes regulating cell proliferation or apoptosis. Cytokine receptors activate mainly the JAK/STAT pathway $[15,16]$ which can either regulate the expression of apoptotic molecules such as Bcl-xL or cell proliferation, depending on the isoforms activated. Chemokines can activate GPCRs which control the PKA/CREB signaling pathway, while growth factors, for example, can activate PI3K/Akt and the MEK/ ERK pathway, via tyrosine receptors, to regulate gene expression [17]. There is considerable cross-talk between these different pathways during the inflammation and healing process.

\section{The immune system in the CNS}

Seminal experiments in the 1920 s demonstrated that, unlike peripheral tissue, engraftment of tumors into the brain parenchyma did not elicit an immune response and thus the tissue graft was not rejected $[18,19]$. This gave rise to the idea that the brain is an immune-privileged organ; an idea which still holds though has been greatly refined. Immune-privilege does not refer to an absolute but rather relative state, and the level of immune-privilege differs between compartments of the brain [20,21].

In the healthy brain, the subarachnoid space, cerebrospinal fluid and vasculature contain circulating leukocytes including dendritic cells, macrophages and $\mathrm{T}$ cells, however the entry of these cells into the brain parenchyma is highly restricted and regulated [21]. The brain parenchyma instead contains the tissue-resident macrophages known as microglia, which are a self-renewing population derived from yolk-sac myeloid precursor cells which invade the CNS between E8.5 and E9.5 in mice [22]. Two groundbreaking studies $[23,24]$ examined microglia dynamics in the healthy, uninjured adult brain using in vivo imaging and unexpectedly found that microglia processes are continuously surveying the brain parenchyma and are the most morphologically plastic cells in the CNS. Additionally, microglia processes were found to contact synapses and this interaction can be modified by neuronal activity $[25,26]$. These studies shifted the view of microglia in the healthy brain from quiescence to active surveillance, and gave rise to the notion that microglia play a role in synaptic physiology. From a surveillance mode, various stimuli can cause the microglia activation which might lead to changes in morphology (for example, from ramified to amoeboid), the release of cytotoxic or neuroprotective factors (such as cytokines and growth factors), alterations in gene and surface receptor expression, and phagocytosis of tissue debris or pathogens [27-29].

Astrocytes are the main resident glial CNS cell population and are the second main source of brain cytokines. Following brain injury, astrocytes are activated and form a reactive astrogliosis, a process important for isolating the injured area and protect the adjacent cells but which is deleterious for neuronal axonal regeneration [30, 31]. Astrocytes also form the glia limitans surrounding the vasculature of the CNS and control the entry of peripheral immune cells into the brain parenchyma [21].

Additionally, injured neurons release factors such as cytokines and neurotransmitters that recruit and activate the other CNS cells involved in neuroinflammation. For example, microglia can be activated by cytokines, by the detection of cellular damages [13] and by neurotransmitters released during injury [32]. Activated microglia release a large array of inflammatory mediators leading also to the recruitment of peripheral macrophages, dendritic cells and $\mathrm{T}$ lymphocytes [14, 33-36] directly from meningeal lymphatic vessels or blood vessels $[37,38]$. Astrocytes and adjacent neurons are also activated in parallel to this cascade leading to further factor release [39] (Fig. 2).

Most of the dialog between these different cell types is due to factors released in the extracellular medium (volume communication) though direct contacts are involved in some key steps. Neurons express most of the receptors associated with the released factors during neuroinflammation and are sensitive to the gazotransmitters or reactive oxygen species released by inflammatory cells. It remains to be determined how the subcellular localization of these receptors is important to modify synaptic activity and whether they have to be present at the modified synapse or if they can affect it at distance.

\section{Synaptoimmunological mechanisms involved in physiological processes}

Initially believed to play a role only during inflammation processes, increasing evidence suggest that immune signals are essential components of normal synaptic functioning, including roles in synaptic plasticity and stability [2].

\section{Synaptic plasticity}

Most synaptic plasticity occurs at glutamatergic synapses, where transmission is mediated by ionotropic receptors (GluN, GluA and GluK) and metabotropic receptors (mGlu). Transporters, expressed on neurons and astrocytes, limit the activation of glutamate receptors. All these receptors are subject to many post-translational modifications, amongst which two of the most important are protein/protein interactions and phosphorylation.

One main role of cytokines in synaptic transmission is their ability to modulate the induction of synaptic 
plasticity. IL-1 $\beta$, IL-2, IL-6, IL-8, IL-18, IFN $\alpha$, IFN $\gamma$ and TNF $\alpha$ have all been shown to be able to inhibit LTP and to induce changes in hippocampal dependent learning and memory tasks [40-42]. LTD can also be inhibited either directly by cytokines like IL-1 $\beta$ or during the inflammation process $[40,43]$. Conversely, under physiological conditions, activation of the JAK2/STAT3 pathway is required for the induction of GluN-dependent LTD in the hippocampus [12]. Further the induction of LTP increases the expression of IL- 6 which acts in a negative feedback manner to limit the magnitude of potentiation $[44,45]$. These results demonstrate that cytokine signaling not only acts in a metaplastic manner to modulate bidirectional changes in synaptic efficacy, but is also part of the physiological mechanism.

The IL- $1 \beta$ receptor has been shown to physically interact with GluN receptors enabling the rapid regulation of GluN activity, via Src-dependent phosphorylation events [46]. The IL-1 $\beta$ receptor can also decrease GluA surface expression [47]. TNF $\alpha$ can promote GluA dependent activity in hippocampal neuron and may induce GluA internalization in striatal GABAergic neurons (reviewed in [48]).

The mechanisms by which cytokines or inflammation alter synaptic function is complex as microglia by themselves can directly facilitate synaptic strength independently of any changes in synaptic activity. Once activated, they can, for example, induce STP via IL-1 $\beta$ dependent mechanisms [49] or LTD in pathological context [50].

In addition to cytokines, major histocompatibility complex (MHC) class I molecules also play a role in modulating the induction of synaptic plasticity. MHC class I molecules are a group of proteins which, within the immune system, translocate cytosolic peptides generated by proteasome-mediated degradation to the cell surface for recognition and subsequent cell elimination by cytotoxic $\mathrm{T}$ cells [51]. MHC class I molecules have also been found to be expressed by neurons in the CNS and localize to synapses, axon terminals and dendrites [52-56]. In the dorsal lateral geniculate nucleus (dLGN), the MHC class I molecule $\mathrm{H} 2-\mathrm{D}^{\mathrm{b}}$ was found to be necessary in limiting the synaptic incorporation of calciumpermeable AMPARs and thus permit the induction of LTD [57]. In the hippocampus, $\beta_{2} \mathrm{M}-/-$ TAP-/- mice (which lack cell surface expression of MHC class I molecules) have a shift in LTP threshold in area CA1, such that low-frequency stimulation, which usually induces LTD, instead causes LTP, and LTP induced by high-frequency stimulation is larger in magnitude $[54,58]$. Finally, MHC class I acts as a negative regulator of synapse density in both the cortex and hippocampal area CA3 [59, 60], which, in area CA3, is the result of a MHC class I-insulin receptor complex which constrains basal insulin receptor signaling [60].
Finally, the complement system, which is part of the innate immune system, and in particular the complement component $\mathrm{C} 3$, could also play a direct role by modulating the efficiency of glutamatergic synaptic transmission in the absence of any inflammation process, by a mechanism not yet explored [61], but that could involve synaptic stripping [62], a process initially defined as the removal of dysfunctional synapses by activated microglia [63].

\section{Structural plasticity}

Organisms are born with an excess number of synapses throughout the CNS, and during development superfluous connections are removed in an experience-dependent manner, a process known as synaptic pruning [64]. Within the CNS synaptic pruning has been extensively studied in the visual system, specifically the LGN and the striate cortex. Initially, LGN neurons receive inputs from multiple retinal ganglion cells (RGCs), however during postnatal development inputs are selectively removed such that eventually each LGN neuron only receives input from one or two RGCs [65]. Additionally, monocular deprivation (MD) during the developmental critical period can lead to reduced responsiveness to the deprived eye in the LGN and striate cortex as deprived eye inputs are weakened [66]. Stevens and colleagues [67] found that the classic complement cascade mediated the elimination of RGC inputs onto LGN neurons via microglia phagocytosis early in postnatal development (P5). A subsequent study found that complement-mediated synaptic pruning in the LGN is regulated by neuronal activity, as inhibiting activity in one eye with TTX increased microglia engulfment of that eye's inputs, while the converse occurred when RGC activity in one eye was stimulated with forskolin [68]. The developing visual system model has also revealed the involvement of other immune pathways in synaptic pruning including purinergic signaling with microglia via the P2Y12 receptor [69] and MHC class I proteins [57].

In the developing hippocampus it has been demonstrated that puncta of the postsynaptic protein PSD-95 are contained within microglia, providing evidence of microgliamediated synaptic pruning. Further, knocking out the microglia-specific fractalkine receptor CX3CR1 resulted in an increased spine density in neonatal mice [70]. It was also found that CX3CR1 KO mice had impaired synapse maturation [70, 71], assayed by measuring the number of release sites per neuron-neuron connection, and thus the authors proposed that that synapse elimination allows for subsequent strengthening of remaining synapses [71]. However, CX3CR1 KO mice were also found to have enhanced IL-1 $\beta$ levels which resulted in a specific impairment of LTP [72], suggesting that the effects of deficient synaptic pruning and synapse maturation observed in CX3CR1 KO mice may not be directly linked. 
It has been proposed that weakened synapses are subsequently 'tagged' with complement proteins to induce microglia phagocytosis [73]. Synapse elimination has been found to occur in the hippocampus following both mGlu- and GluN-dependent LTD [74-79]. Thus, future studies should seek to directly examine whether there is an interaction between synaptic depression and microglia phagocytosis.

In the adult brain, activation of microglia leads to the displacement of inhibitory synapses from the soma of neurons and is neuroprotective by a mechanism involving GluN activation [80-82]. Synaptic stripping by microglia participates in network remodeling but its exact role in pathology remains to be fully demonstrated [83].

Synaptic pruning can also occur independently of any physical interaction with glia. This mechanism has been well described for neurons with axon injury and occurs concomitantly with glial cell activation. Astrocytes as well as microglia release numerous factors (cytokines, chemokines, thrombospondins, etc.) that directly influence synapse integrity $[84,85]$.

Though research has mainly focused on microglia in relation to synapse elimination, recent advances have shed light on the role microglia also play in spine formation. The generation of CX3CR1-CreER mice allowed Parkhurst and colleagues [86] to conditionally deplete microglia or knockout microglial BDNF. Subsequent in vivo two photon imaging revealed that these manipulations impaired spine formation in the motor cortex following motor learning. Furthermore, in vivo two photon imaging of the somatosensory cortex of developing mice revealed that microglia contact of dendrites frequently led to spine filipodia formation [87]. Therefore, it appears that microglia can cause bidirectional modifications of dendrite spine structure but the underlying mechanisms (see also the review of Kettenman et al. [63]) involved in this process need more investigation by future studies.

\section{Synaptic scaling}

At glutamatergic synapses, when extended periods of elevated or depressed neuronal activity occur, homeostatic mechanisms can be activated. They alter strength across all synapses to return activity to an optimal range, a process known as synaptic scaling [88]. Chronic (24-48 h) blockade of neuronal action potential firing or glutamatergic synaptic transmission results in a large increase in mEPSC amplitude (a putative measure of post-synaptic sensitivity to glutamate) as well as the number of surface AMPARs [89]. Stellwagen and Malenka [90] found that TNF $\alpha$ is both necessary and sufficient for scaling up of post-synaptic AMPARs. Interestingly, although both neurons and glia are capable of producing TNF $\alpha$, the authors found that it is the glia-released TNF $\alpha$ that is critical for scaling up of synapses. A subsequent study found that $\beta 3$ integrins are also necessary for scaling up of synapses, and that TNF $\alpha$ application increases the surface expression of $\beta 3$ integrin [91]. Further, the authors found that levels of $\beta 3$ integrin control surface AMPARs and thus mEPSC amplitude, suggesting a model in which glial cells, in response to reduce network activity, release TNF $\alpha$ leading to an increase in $\beta 3$ integrin surface expression and subsequent accumulation of AMPARs at the synapse [92].

To address the physiological significance of TNF $\alpha$ meditated synaptic scaling, Kaneko and colleagues [93] examined visual system. In the striate cortex, similar to the hippocampus, LTP was normal in TNF $\alpha-/-$ mice, however scaling up of AMPAR-mediated mEPSCs was absent. In vivo, $\mathrm{TNF} \alpha-/-$ mice had impaired ocular dominance plasticity following MD, specifically having a complete deficit in the increase of non-deprived eye cortical response, despite a normal decrease in the cortical response for the deprived eye. Thus, synaptoimmunological factors are critical for both phases of ocular dominance plasticity, with the complement cascade mediating the loss of cortical responsiveness to the deprived eye, while TNFa mediates the compensatory, homeostatic increase in non-deprived eye cortical responsiveness.

\section{Synaptoimmunological mechanisms involved in acute brain disorders}

Causes of brain disorders associated with neuroinflammation and synaptic function alteration are numerous. Some disorders appear following a brief episode associated an infection, whereas others have either undefined causes or genetic origins. The mechanisms involved in synaptic function alteration are dependent of the nature of the cause.

\section{Acute infection}

Following a systemic or a direct brain infection, an immunological response is triggered and coordinated by the brain and the immune system. In general acute and longterm infections of the brain and spinal cord are produced mostly by traumatic injury, parasites, intoxication and systemic infectious diseases caused by viruses, bacteria, fungi and parasites, which penetrate the central nervous system. A common and potentially life-threatening form of generalized inflammatory response is sepsis, which is characterized by an overreaction of the immune system. The pathophysiology of the sepsis is highly complex and affects all types of brain cells and brain functions (for a comprehensive review of sepsis and brain dysfunctions see [94]).

Brain viral infections (e.g., by influenza, HIV, Herpes, West Nile virus (WNV) has been reported to both directly and indirectly (by promoting neuroinflammation) affect synaptic functions, leading to cognitive impairment [95-97]. For example, a recent study found that 
synapse loss in a mouse model of WNV infection is driven by the activation of the classical complement cascade in the hippocampus [62]. Further, various viral proteins reduce voltage dependent calcium channel [98] or GluA function [99]. Interestingly some viral effects require NMDAR activation or alteration through PKA and PKC dependent mechanisms (for a review see [96]). Additionally, certain viral dependent synapse impairments are also explained by the ability of viruses to indirectly induce the expression or interfere with the function of proteins associated with synaptic impairment like A $\beta$ or APP [100].

\section{Acute brain injuries without pathogen}

Acute brain injuries are often associated with an inflammation response in the absence of any pathogen, a mechanism termed sterile inflammation, which can be caused by multiple different events such as mechanical trauma, ischemia, stress, alcohol etc.. Three of the main pathologies arising from these sterile injuries are Traumatic Brain Injuries (TBI), epilepsy and stroke, pathologies which share common cell death mechanisms [101]. This inflammation has been associated both with the worsening of the pathologies and with the repair phase [101-104], but the mechanisms involved in the alteration of synapse functions could be pathology-specific.

Synapses, by their intrinsic complex architecture including PSD and adhesion molecules, contribute largely to the diffusion of mechanical trauma during TBI [105] suggesting that inflammation dependent alteration of synapse integrity may be directly involved in the severity of the pathology.

Epilepsy and inflammation are strongly linked (reviewed in [106]). Synapse elimination, sprouting and changes in synaptic strength are key features of this pathology. Inflammation impacts epilepsy directly by modulating synaptic activity via altered protein expression through the activation of the NFKB pathway or by altering synaptic channel activity via phosphorylation cascades [107]. Computational network modeling has also predicted that TNF $\alpha$ release by glia following inflammation can lead to epileptogenesis through scaling up of synapses [108].

The effect of sterile inflammation on synaptic function may also depend of changes in neuronal environment during pathology. Thus TNF $\alpha$ enhances LTP in the context of ischemia via a p38 MAPK dependent mechanism, whereas it blocks LTP in the physiological context [109]. The alteration of glutamatergic transmission disappears when inflammation resolved [40].

Another prominent cause of brain inflammation is alcohol abuse, as illustrated by the phenomenon of "binge drinking" [110]. It is well documented that alcohol directly affects glutamate receptors (GluRs) and other families of receptors [111-117] as well as synaptic plasticity [118].
Critically several studies in humans $[113,119,120]$ and animals [121-124] have provided strong evidence that the effect of alcohol abuse on GluRs harms brain development, synaptic refinement and impairs cognitive functions.

\section{Synaptoimmunological dysregulation in neurodegenerative/autoimmune brain disorders}

In recent years, the classical dichotomy between inflammation and neurodegeneration has been challenged by evidence suggesting that both aspects are interconnected both in neurodegenerative diseases, including Alzheimer's disease (AD) and Parkinson's disease (PD), and in traditional neuroinflammatory disorders, such as multiple sclerosis (MS) $[125,126]$. Growing experimental evidence suggests that synapses may be the locus for abnormalities underlying these diseases. Indeed, perturbations in the induction, maintenance or reversal of LTP and LTD are a common thread in the different brain disease models [127, 128], as well as in human pathologies associated with inflammation [129]. However, there are disease-specific mechanisms of how synapse structure and function are precisely affected in each disorder. It is thus reasonable to postulate that the combination of abnormal expression of immune mediators along with other disease-specific features might contribute to the distinct etiopathogenesis of different conditions.

\section{Multiple sclerosis/EAE}

Multiple sclerosis (MS), especially its relapsing-remitting form, is a complex immune-mediated disease [130]. The neuroinflammatory milieu that typically characterizes MS profoundly impacts the capability of neuronal systems to express normal plasticity, possibly leading to a state of decreased homeostatic reserve with negative consequences on cognitive performances. Inflammation-induced synaptic dysfunction appears in the very early phases of MS patients and in the experimental autoimmune encephalomyelitis (EAE), a well-established mouse model of multiple sclerosis. Accordingly, it was recently shown that intermittent (iTBS) or continuous theta burst stimulations (cTBS), delivered through a transcranial magnetic stimulation (TMS) device, modulate the expression of cortical plasticity in the acute inflammatory phases of MS patients. In general, LTP was always favored over LTD in response to repetitive synaptic activation in MS brains, and this effect was directly correlated with IL-1 $\beta$ levels in the CSF [129]. Similar results were also observed in hippocampal slices from EAE mouse, in which the facilitation of CA1-LTP was also mediated via enhanced IL-1 $\beta$ released from CD3+ T lymphocyte infiltrates or activated microglia, clearly detectable in the EAE hippocampus [43, 131]. Remarkably, preventive or pharmacological strategies restraining pro-inflammatory cytokines and oxidative stress were able to rescue synaptic alterations in the EAE model [132, 133]. 


\section{Alzheimer's disease}

$\mathrm{AD}$ is a chronic neurodegenerative disease characterized by progressive neuronal loss and cognitive decline. Oligomeric amyloid $\beta(\mathrm{oA} \beta)$ is implicated in the pathogenesis of $\mathrm{AD}$ and disrupts synaptic plasticity through numerous mechanisms (Fig. 3) [127]. Inflammatory features including activation and proliferation of glia and expression of mediators such as IL-1, IL-6, and TNF $\alpha[133,134]$ have been clearly detected in the brain, CSF and peripheral blood of AD patients. These molecules are linked to immune cell activation and strongly affect LTP, even though the relation between oA $\beta$ and inflammation remains unclear. Regardless, it is conceivable that the 'early' loss of

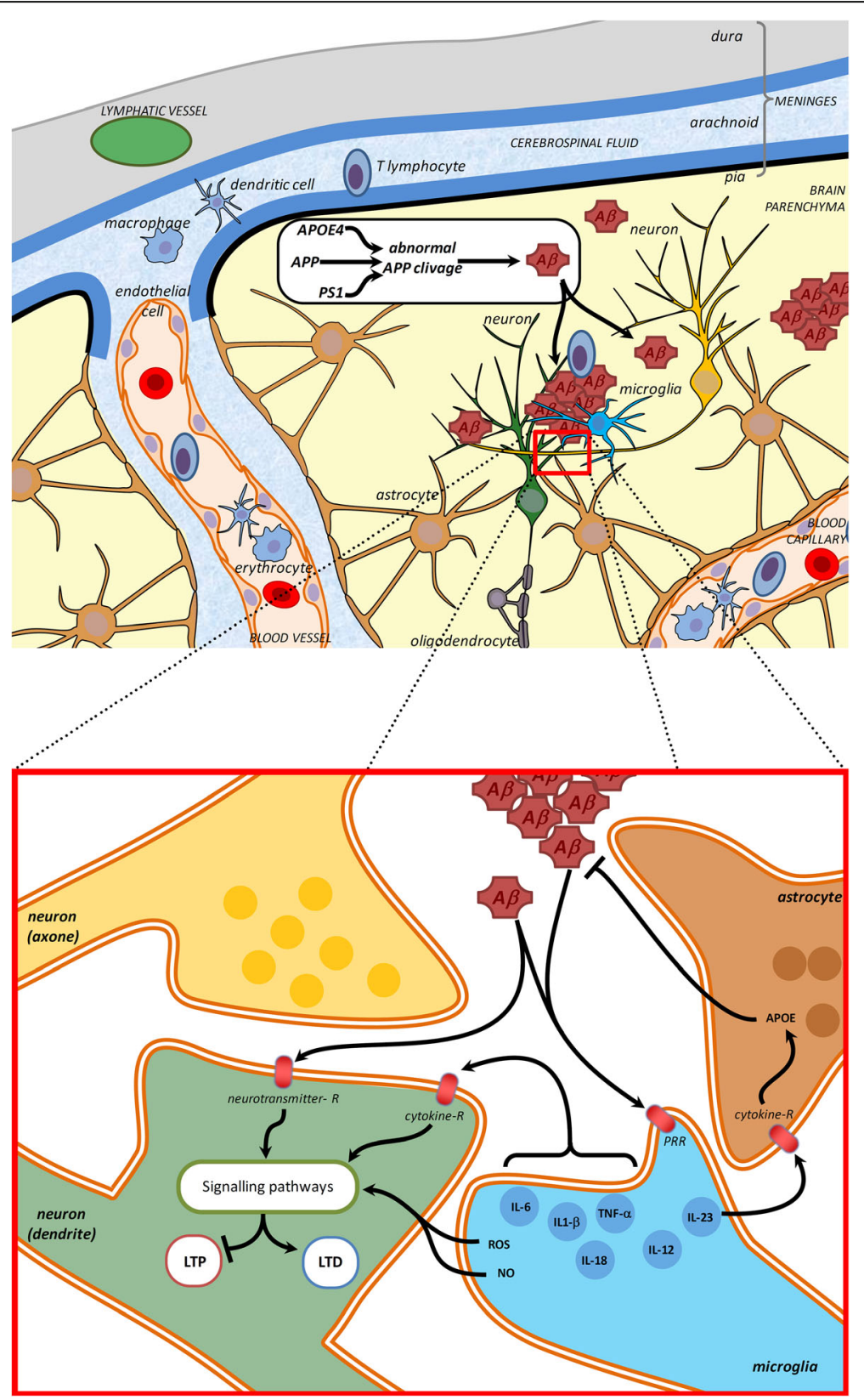

Fig. 3 Signaling pathways in inflammation / immune response and how genetic risk factors for (e.g. AD) may impact via these pathways. Oligomeric or aggregate of amyloid beta peptides $(A \beta)$, as occurring during Alzheimer's disease, are detected by pattern recognition receptor (PRR) like Toll like receptors. In microglia they stimulate the production and release of cytokines such as interleukins (IL). These interleukins are detected by astrocytes and neurons, where they stimulate signaling pathways that interfere directly with the signaling pathways activated during synaptic plasticity, inducing deficits in LTP or exacerbated LTD. Aß peptides can also interfere directly with neurotransmitter receptors (Glutamate receptors (GluN, mGlu) or acetylcholine receptors) leading to abnormal neurotransmission. (top scheme, cerebral structure inspired from [37]) 
hippocampal LTP observed in AD represents a downstream effect of the presence of both oA $\beta$ and ongoing neuroinflammation.

Among cytokines, TNF $\alpha$ and IL-1 $\beta$ have been shown to mediate the detrimental effects of oA $\beta$ on LTP. Indeed, suppression of LTP by oA $\beta$ was absent in mutant mice null for TNF receptor type 1 and was prevented by the monoclonal antibody infliximab, the TNF peptide antagonist, and thalidomide, the inhibitor of TNF $\alpha$ production [135]. Further, intracerebroventricular administration of interleukin 1 receptor antagonist (IL-1ra) rescued posttetanic potentiation impairment following injection of oA $\beta$ peptide [136].

To further support a role for inflammation in AD, evidence from epidemiologic studies and clinical trials suggest that non-steroidal anti-inflammatory agents (NSAIDs) exert neuroprotection in AD. Accordingly, two selective COX-2 inhibitors were effective in preventing the disruption of LTP by synthetic soluble A $\beta$ [137].

An emerging issue is how microglia, which physiologically control synapse function and plasticity, contribute to AD pathogenesis. Recent evidence suggests that failure of signaling required for maintaining a 'resting' microglial phenotype, likely important for preserving surveillance functions, might have profound consequences on synaptic activity. For instance, numerous studies have found that $\mathrm{A} \beta$ increases microglia activation and the release of cytokines which impair LTP, and that inhibiting microglia activation can prevent the block of LTP induction by $\mathrm{A} \beta$ (for a recent review see [138]). Further the loss of synapses, a hallmark feature of $\mathrm{AD}$, has been linked to microglia phagocytic activity. A recent study found that synapse loss in the hippocampus in the early stages of a mouse model of $\mathrm{AD}$, as well as following direct infusion of oA $\beta$, was mediated by the complement cascade $(\mathrm{C} 1 \mathrm{q}$, C3 and CR3). Thus the authors suggest that microgliamediated synapse loss early in $\mathrm{AD}$ may be due to a pathological reactivation of a developmental program of synaptic pruning [139].

\section{Parkinson's disease}

Similar to the synaptotoxic role played by oA $\beta$ in $A D$, extracellular alpha-synuclein oligomers also modulate synaptic transmission and impair LTP [140]. However, results obtained in this study are questionable since alpha-synuclein oligomers were applied at supraphysiological concentrations. Aggregation of $\alpha$-synuclein triggers the release of TNF $\alpha$ and IL- $1 \beta$ from microglia, and this might lead to its toxic effects on dopaminergic cells [141]. Several authors found elevated TNF, IL-1, IL-6, IL-2 and upregulation of MHC molecules in the striatum and CSF of PD patients [142], thus supporting the hypothesis that immune response is a pathogenic mechanism underlying PD. Notably, treatment with the flavonoid baicalein decreased upregulation of TNF $\alpha$ and IL-1 $\beta$ and normalized striatal glutamatergic transmission in a rodent model of PD [143]. Presence of a persistent active inflammatory process in $\mathrm{PD}$ patients might contribute to the impairment of physiological synaptic plasticity at corticostriatal synapses. This, in turn, might lead to disruption of signaling pathways within the basal ganglia neuronal network [144] as the basis of PD symptomatology.

Further studies are still required to clarify the precise role that cytokines might play in striatal synaptic plasticity in physiological and pathological conditions.

\section{Therapeutic implications and conclusions}

During the course of an immune attack, release of pro-inflammatory cytokines is temporary and normally controlled by anti-inflammatory mechanisms, representing an adaptive and regulated response of the brain to immune signals. Conversely, when the immune challenge becomes prolonged and/or uncontrolled, the consequent inflammatory response might lead to pathological conditions.

Despite remarkable progress in the knowledge of cell signaling in neuroimmunology, to date several key questions still need to be addressed. For example, whereas immune protein function has been well characterized within the immune cells, not as much is known about how immune proteins exert their non-immune role to influence signaling pathways and gene expression engaged in synaptic plasticity in neurons. Next, it is still unclear how pathways are precisely activated by cytokines in target cells within a physiological or pathological setting. One critical issue might relate to the different concentrations of cytokines between the in vitro and in vivo condition. In fact, cytokines are generally used within the nanomolar range in vitro, whereas their in vivo levels in the brain fall within the picomolar range, making it difficult to define the realistic cytokine exposure at the synaptic level following different stimuli.

Considering the importance of immune mechanisms on neurotransmitter systems and brain circuitries relevant to neuropsychiatric diseases, a better understanding of brain-immune system interactions will hopefully provide specific biomarkers to measure the status of the neuroimmunological response, as well as novel neuroimmunetargeted therapeutics.

\section{Abbreviations}

AD: Alzheimer's disease; AMPAR: AMPA receptor; APP: Amyloid precursor protein; CaMKII: Ca2+/ calmodulin-dependent kinase II; CNS: Central nervous system; COX-2: Cyclooxygenase 2; CTBS: Continuous theta burst stimulations; DAMPs: Damage associated molecular patterns; dLGN: Dorsal lateral geniculate nucleus; EAE: Experimental autoimmune encephalomyelitis; eEF2K: Eucariotic elongation factor 2 kinase; GluA: Glutamate AMPA; GluK: Glutamate kainate; GluN: Glutamate NMDA; GluRs: Glutamate receptors; GPCRs: G protein coupled 
receptors; IFNa: Interferon a; IFNy: Interferon $\gamma ;$ IL-18: Interleukin 18; IL-1B: Interleukin 13; IL-2: Interleukin 2; IL-6: Interleukin 6; IL-8: Interleukin 8; ITBS: Intermittent theta burst stimulations; LTD: Long term depression; LTP: Long term potentiation; MD: Monocular deprivation; mEPSC: Miniature excitatory post synaptic current; mGlu: Metabotropic glutamate; MHC: Major histocompatibility complex; MS: Multiple sclerosis; NMDAR: NMDA receptor; NSAIDs: Non-steroidal anti-inflammatory agents; $O A \beta$ : Oligomeric amyloid $\beta$, PAMPs: Pathogen associated molecular patterns; PD: Parkinson's disease; PI3K: Phosphoinositide-3-kinase; PKA: Protein kinase A; PKC: Protein kinase C; PLC: Phospholipase C; PRR: Pattern recognition receptors; RGCs: Retinal ganglion cells; STD: Short term depression; STP: Short term potentiation; TBI: Traumatic brain injury; TLRs: Toll-like receptors; TNF: Tumor necrosis factor; TNFa: Tumor necrosis factor a; TTX: Tetrodotoxin; WNV: West Nile virus

\section{Acknowledgements}

Not applicable.

\section{Funding}

SP is supported by Medical Research Coucil (MR/K023098/1) and is holder of the chair of excellence "Synaptic Plasticity" funded by Région Hauts de France.

\section{Availability of data and materials}

Not applicable.

\section{Authors' contributions}

All authors contributed to writing the manuscript. All authors read and approved the final manuscript.

\section{Competing interests}

None of the authors have any competing interests in the manuscript.

\section{Consent for publication}

Not applicable.

\section{Ethics approval and consent to participate}

Not applicable.

\section{Publisher's Note}

Springer Nature remains neutral with regard to jurisdictional claims in published maps and institutional affiliations.

\section{Author details \\ ${ }^{1}$ Department of Biology, University of Rome Tor Vergata, 00133 Rome, Italy. ${ }^{2}$ Pharmacology of Synaptic Disease Lab, European Brain Research Institute, 00143 Rome, Italy. ${ }^{3}$ Department of Physiology, University of Toronto, and Lunenfeld-Tanenbaum Research Institute, Mount Sinai Hospital, Toronto, ON, Canada. ${ }^{4}$ Centre for Synaptic Plasticity, School of Physiology, Pharmacology \& Neuroscience, University of Bristol, Bristol, UK. ${ }^{5}$ PROTECT, INSERM, Université Paris Diderot, Sorbonne Paris Cité, Paris, France. ${ }^{6}$ Centre for the Developing Brain, King's College, St Thomas' Campus, London, UK. ${ }^{7}$ INSERM-ERi 24 (GRAP), Centre Universitaire de Recherche en Santé, Université de Picardie} Jules Verne, Amiens, France.

Received: 12 April 2017 Accepted: 13 June 2017

\section{Published online: 20 June 2017}

\section{References}

1. Boulanger LM. Immune proteins in brain development and synaptic plasticity. Neuron. 2009:64(1):93-109.

2. Marin I, Kipnis J. Learning and memory ... and the immune system. Learn Mem. 2013;20(10):601-6.

3. Collingridge GL, et al. Long-term depression in the CNS. Nat Rev Neurosci. 2010;11(7):459-73.

4. Nicoll RA, Roche KW. Long-term potentiation: peeling the onion. Neuropharmacology. 2013;74:18-22

5. Feldman DE. The spike-timing dependence of plasticity. Neuron. 2012;75(4):556-71.

6. Borbely E, Scheich B, Helyes Z. Neuropeptides in learning and memory. Neuropeptides. 2013;47(6):439-50.

7. van den Pol AN. Neuropeptide transmission in brain circuits. Neuron. 2012;76(1):98-115.
8. Casillas-Espinosa PM, Powell KL, O'Brien TJ. Regulators of synaptic transmission: roles in the pathogenesis and treatment of epilepsy. Epilepsia. 2012;53(Suppl 9):41-58.

9. Baudry $\mathrm{M}$, et al. Multiple cellular cascades participate in long-term potentiation and in hippocampus-dependent learning. Brain Res. 2015;1621:73-81.

10. Sacktor TC. How does PKMzeta maintain long-term memory? Nat Rev Neurosci. 2011;12(1):9-15.

11. Bliim N, et al. Transcriptional regulation of long-term potentiation. Neurogenetics. 2016;17(4):201-10

12. Nicolas CS, et al. The Jak/STAT pathway is involved in synaptic plasticity. Neuron. 2012;73(2):374-90.

13. Kigerl KA, et al. Pattern recognition receptors and central nervous system repair. Exp Neurol. 2014;258:5-16.

14. Downes CE, Crack PJ. Neural injury following stroke: are Toll-like receptors the link between the immune system and the CNS? Br J Pharmacol. 2010;160(8):1872-88.

15. O'Shea JJ, Murray PJ. Cytokine signaling modules in inflammatory responses. Immunity. 2008;28(4):477-87.

16. Delgoffe GM, Murray PJ, Vignali DA. Interpreting mixed signals: the cell's cytokine conundrum. Curr Opin Immunol. 2011;23(5):632-8.

17. Bezbradica JS, Medzhitov R. Integration of cytokine and heterologous receptor signaling pathways. Nat Immunol. 2009;10(4):333-9.

18. Shirai $Y$. On the transplantation of the rat sarcoma in adult heterogenous animals. Jap Med World. 1921;1:14-5.

19. Murphy JB, Sturm E. Conditions determining the transplantability of tissues in the brain. J of Exp Medicine. 1923;38(2):183-97.

20. Galea I, Bechmann I, Perry VH. What is immune privilege (not)? Trends Immunol. 2007;28(1):12-8

21. Engelhardt $B$, Vajkoczy $P$, Weller RO. The movers and shapers in immune privilege of the CNS. Nat Immunol. 2017;18(2):123-31.

22. Ginhoux F, et al. Fate mapping analysis reveals that adult microglia derive from primitive macrophages. Science. 2010;330(6005):841-5.

23. Davalos $D$, et al. ATP mediates rapid microglial response to local brain injury in vivo. Nat Neurosci. 2005:8(6):752-8.

24. Nimmerjahn A, Kirchhoff F, Helmchen F. Resting microglial cells are highly dynamic surveillants of brain parenchyma in vivo. Science. 2005;308(5726):1314-8

25. Wake $\mathrm{H}$, et al. Resting microglia directly monitor the functional state of synapses in vivo and determine the fate of ischemic terminals. J Neurosci. 2009:29(13):3974-80.

26. Tremblay ME, Lowery RL, Majewska AK. Microglial interactions with synapses are modulated by visual experience. PLoS Biol. 2010;8(11):e1000527.

27. Suzumura A. Neuron-microglia interaction in neuroinflammation. Curr Protein Pept Sci. 2013;14(1):16-20.

28. Czeh M, Gressens P, Kaindl AM. The yin and yang of microglia. Dev Neurosci. 2011;33(3-4):199-209.

29. Tremblay ME, et al. The role of microglia in the healthy brain. J Neurosci. 2011;31(45):16064-9.

30. Li C, et al. Astrocytes: implications for neuroinflammatory pathogenesis of Alzheimer's disease. Curr Alzheimer Res. 2011;8(1):67-80.

31. Pekny M, Pekna M. Astrocyte reactivity and reactive astrogliosis: costs and benefits. Physiol Rev. 2014;94(4):1077-98.

32. Kaindl $\mathrm{AM}$, et al. Activation of microglial $\mathrm{N}$-methyl-D-aspartate receptors triggers inflammation and neuronal cell death in the developing and mature brain. Ann Neurol. 2012:72(4):536-49.

33. Wraith DC, Nicholson LB. The adaptive immune system in diseases of the central nervous system. J Clin Invest. 2012;122(4):1172-9.

34. Pedemonte $E_{\text {, et }}$ al. Mechanisms of the adaptive immune response inside the central nervous system during inflammatory and autoimmune diseases. Pharmacol Ther. 2006;111(3):555-66.

35. Ransohoff RM, Engelhardt B. The anatomical and cellular basis of immune surveillance in the central nervous system. Nat Rev Immunol. 2012;12(9):623-35.

36. London A, Cohen M, Schwartz M. Microglia and monocyte-derived macrophages: functionally distinct populations that act in concert in CNS plasticity and repair. Front Cell Neurosci. 2013;7:34

37. Louveau A, et al. Structural and functional features of central nervous system lymphatic vessels. Nature. 2015;523(7560):337-41.

38. Louveau A, Harris TH, Kipnis J. Revisiting the Mechanisms of CNS Immune Privilege. Trends Immunol. 2015;36(10):569-77.

39. Heneka MT, Kummer MP, Latz E Innate immune activation in neurodegenerative disease. Nat Rev Immunol. 2014;14(7):463-77. 
40. Riazi K, et al. Microglia-dependent alteration of glutamatergic synaptic transmission and plasticity in the hippocampus during peripheral inflammation. J Neurosci. 2015;35(12):4942-52.

41. Lynch MA. Neuroinflammatory changes negatively impact on LTP: A focus on IL-1beta. Brain Res. 2015;1621:197-204.

42. Patterson SL. Immune dysregulation and cognitive vulnerability in the aging brain: Interactions of microglia, IL-1 beta, BDNF and synaptic plasticity. Neuropharmacology. 2015;96(Pt A):11-8.

43. Nisticò $R$, et al. Inflammation subverts hippocampal synaptic plasticity in experimental multiple sclerosis. PLoS One. 2013;8(1):e54666.

44. Jankowsky JL, Derrick BE, Patterson PH. Cytokine responses to LTP induction in the rat hippocampus: a comparison of in vitro and in vivo techniques. Learn Mem. 2000;7(6):400-12.

45. Balschun D, et al. Interleukin-6: a cytokine to forget. FASEB J. 2004;18(14):1788-90.

46. Gardoni F, et al. Distribution of interleukin-1 receptor complex at the synaptic membrane driven by interleukin-1 beta and NMDA stimulation. $J$ Neuroinflammation. 2011:8(1):14

47. Lai AY, et al. Interleukin-1 beta modulates AMPA receptor expression and phosphorylation in hippocampal neurons. J Neuroimmunol. 2006;175(1-2):97-106.

48. Vezzani A, Viviani B. Neuromodulatory properties of inflammatory cytokines and their impact on neuronal excitability. Neuropharmacology. 2015;96(Pt A):70-82.

49. Clark AK, et al. Selective activation of microglia facilitates synaptic strength J Neurosci. 2015;35(11):4552-70.

50. Zhang J, et al. Microglial CR3 activation triggers long-term synaptic depression in the hippocampus via NADPH oxidase. Neuron. 2014;82(1):195-207.

51. Hewitt EW. The MHC class I antigen presentation pathway: strategies for viral immune evasion. Immunology. 2003;110(2):163-9.

52. Corriveau RA, Huh GS, Shatz CJ. Regulation of class I MHC gene expression in the developing and mature CNS by neural activity. Neuron. 1998;21(3):505-20.

53. Lidman O, Olsson T, Piehl F. Expression of nonclassical MHC class I (RT1-U) in certain neuronal populations of the central nervous system. Eur J Neurosci. 1999:11(12):4468-72

54. Huh GS, et al. Functional requirement for class I MHC in CNS development and plasticity. Science. 2000;290(5499):2155-9.

55. Goddard CA, Butts DA, Shatz CJ. Regulation of CNS synapses by neuronal MHC class I. Proc Natl Acad Sci. 2007;104(16):6828-33.

56. Needleman LA, et al. MHC class I molecules are present both pre-and postsynaptically in the visual cortex during postnatal development and in adulthood. Proc Natl Acad Sci. 2010;107(39):16999-7004.

57. Lee $\mathrm{H}$, et al. Synapse elimination and learning rules co-regulated by MHC class I H2-Db. Nature. 2014;509(7499):195-200

58. Nelson PA, et al. MHC class I immune proteins are critical for hippocampusdependent memory and gate NMDAR-dependent hippocampal long-term depression. Learn Mem. 2013;20(9):505-17.

59. Glynn $\mathrm{MW}$, et al. $\mathrm{MHCl}$ negatively regulates synapse density during the establishment of cortical connections. Nat Neurosci. 2011;14(4):442-51.

60. Dixon-Salazar TJ, et al. MHC class I limits hippocampal synapse density by inhibiting neuronal insulin receptor signaling. J Neurosci. 2014;34(35):11844-56.

61. Perez-Alcazar $M$, et al. Altered cognitive performance and synaptic function in the hippocampus of mice lacking C3. Exp Neurol. 2014;253:154-64.

62. Vasek MJ, et al. A complement-microglial axis drives synapse loss during virus-induced memory impairment. Nature. 2016;534(7608):538-43.

63. Kettenmann H, Kirchhoff F, Verkhratsky A. Microglia: new roles for the synaptic stripper. Neuron. 2013;77(1):10-8.

64. Hua JY, Smith SJ. Neural activity and the dynamics of central nervous system development. Nat Neurosci. 2004;7(4):327-32.

65. Hooks BM, Chen C. Distinct roles for spontaneous and visual activity in remodeling of the retinogeniculate synapse. Neuron. 2006;52(2): 281-91.

66. Wiesel TN. The postnatal development of the visual cortex and the influence of environment. Biosci Rep. 1982;2(6):351-77.

67. Stevens B, et al. The classical complement cascade mediates CNS synapse elimination. Cell. 2007;131(6):1164-78

68. Schafer DP, et al. Microglia sculpt postnatal neural circuits in an activity and complement-dependent manner. Neuron. 2012;74(4):691-705.

69. Sipe $\mathrm{GO}$, et al. Microglial P2Y12 is necessary for synaptic plasticity in mouse visual cortex. Nat Commun. 2016;7:2-15.

70. Paolicelli RC, et al. Synaptic pruning by microglia is necessary for normal brain development. Science. 2011;333(6048):1456-8.
71. Zhan Y, et al. Deficient neuron-microglia signaling results in impaired functional brain connectivity and social behavior. Nat Neurosci. 2014:17(3):400-6.

72. Rogers JT, et al. CX3CR1 deficiency leads to impairment of hippocampal cognitive function and synaptic plasticity. J Neurosci. 2011;31(45):16241-50.

73. Stephan AH, Barres BA, Stevens B. The complement system: an unexpected role in synaptic pruning during development and disease. Annu Rev Neurosci. 2012;35:369-89.

74. Nagerl UV, et al. Bidirectional activity-dependent morphological plasticity in hippocampal neurons. Neuron. 2004;44(5):759-67.

75. Shinoda Y, et al. Repetition of mGluR-dependent LTD causes slowly developing persistent reduction in synaptic strength accompanied by synapse elimination. Brain Res. 2005;1042(1):99-107.

76. Kamikubo Y, et al. Long-lasting synaptic loss after repeated induction of LTD: independence to the means of LTD induction. Eur J Neurosci. 2006;24(6):1606-16.

77. Ramiro-Cortés $Y$, Israely I. Long lasting protein synthesis-and activitydependent spine shrinkage and elimination after synaptic depression. PLoS One. 2013:8(8):e71155.

78. Wiegert JS, Oertner TG. Long-term depression triggers the selective elimination of weakly integrated synapses. Proc Natl Acad Sci U S A. 2013;110(47):E4510-9.

79. Hasegawa $\mathrm{S}$, et al. Dendritic spine dynamics leading to spine elimination after repeated inductions of LTD. Sci Rep. 2015;5(7707):1-6.

80. Chen Z, et al. Microglial displacement of inhibitory synapses provides neuroprotection in the adult brain. Nat Commun. 2014;5:4486.

81. Chen Z, Trapp BD. Microglia and neuroprotection. J Neurochem. 2016;136(Suppl 1):10-7.

82. Trapp BD, et al. Evidence for synaptic stripping by cortical microglia. Glia. 2007;55(4):360-8.

83. Perry $\mathrm{VH}, \mathrm{O}^{\prime} \mathrm{C}$ - $\mathrm{nnor} \mathrm{V}$. The role of microglia in synaptic stripping and synaptic degeneration: a revised perspective. ASN Neuro. 2010;2(5):e00047.

84. Delpech JC, et al. Microglia in neuronal plasticity: Influence of stress. Neuropharmacology. 2015;96(Pt A):19-28.

85. Tyzack GE, et al. Astrocyte response to motor neuron injury promotes structural synaptic plasticity via STAT3-regulated TSP-1 expression. Nat Commun. 2014;5:4294.

86. Parkhurst CN, et al. Microglia promote learning-dependent synapse formation through brain-derived neurotrophic factor. Cell. 2013;155(7):1596-609.

87. Miyamoto A, et al. Microglia contact induces synapse formation in developing somatosensory cortex. Nat Commun. 2016;7:12540.

88. Turrigiano GG, et al. Activity-dependent scaling of quantal amplitude in neocortical neurons. Nature. 1998;391(6670):892-6.

89. Turrigiano GG. The dialectic of Hebb and homeostasis. Phil Trans R Soc B. 2017;372(1715):20160258.

90. Stellwagen D, Malenka RC. Synaptic scaling mediated by glial TNF-a. Nature. 2006:40(7087):1054-9

91. Cingolani LA, et al. Activity-dependent regulation of synaptic AMPA receptor composition and abundance by $\beta 3$ integrins. Neuron. 2008;58(5):749-62.

92. Aizenman CD, Pratt KG. There's more than one way to scale a synapse. Neuron. 2008:58(5):651-3.

93. Kaneko $\mathrm{M}$, et al. Tumor necrosis factor-a mediates one component of competitive, experience-dependent plasticity in developing visual cortex. Neuron. 2008;58(5):673-80

94. Sonneville $\mathrm{R}$, et al. Understanding brain dysfunction in sepsis. Ann Intensive Care. 2013;3(1):15

95. Wang GF, Li W, Li K. Acute encephalopathy and encephalitis caused by influenza virus infection. Curr Opin Neurol. 2010;23(3):305-11.

96. De Chiara $\mathrm{G}$, et al. Infectious agents and neurodegeneration. Mol Neurobiol. 2012:46(3):614-38.

97. Jurgens HA, Amancherla K, Johnson RW. Influenza infection induces neuroinflammation, alters hippocampal neuron morphology, and impairs cognition in adult mice. J Neurosci. 2012;32(12):3958-68.

98. Brask J, et al. Changes in calcium currents and GABAergic spontaneous activity in cultured rat hippocampal neurons after a neurotropic influenza A virus infection. Brain Res Bull. 2001:55(3):421-9.

99. Brask J, et al. Effects on synaptic activity in cultured hippocampal neurons by influenza A viral proteins. J Neuro-Oncol. 2005;11(4):395-402.

100. Piacentini R, et al. HSV-1 promotes Ca2+-mediated APP phosphorylation and Abeta accumulation in rat cortical neurons. Neurobiol Aging. 2011;32(12):2323 e13-26.

101. Liou AK, et al. To die or not to die for neurons in ischemia, traumatic brain injury and epilepsy: a review on the stress-activated signaling pathways and apoptotic pathways. Prog Neurobiol. 2003;69(2):103-42. 
102. Angeloni $C$, et al. Traumatic brain injury and NADPH oxidase: a deep relationship. Oxidative Med Cell Longev. 2015;2015:370312.

103. Aertker BM, Bedi S, Cox CS Jr. Strategies for CNS repair following TBI. Exp Neurol. 2016;275(Pt 3):411-26.

104. Corps KN, Roth TL, McGavern DB. Inflammation and neuroprotection in traumatic brain injury. JAMA Neurol. 2015;72(3):355-62

105. Hemphill MA, et al. Traumatic brain injury and the neuronal microenvironment: a potential role for neuropathological mechanotransduction. Neuron. 2015;85(6):1177-92.

106. Vezzani A, et al. Epilepsy and brain inflammation. Exp Neurol. 2013;244:11-21.

107. Vezzani A, et al. The role of inflammation in epilepsy. Nat Rev Neurol. 2011;7(1):31-40.

108. Savin C, Triesch J, Meyer-Hermann M. Epileptogenesis due to glia-mediated synaptic scaling. J R Soc Interface. 2009;6(37):655-68.

109. Wall AM, et al. Tumor necrosis factor-alpha potentiates long-term potentiation in the rat dentate gyrus after acute hypoxia. J Neurosci Res. 2015;93(5):815-29.

110. Petit $\mathrm{G}$, et al. Binge drinking in adolescents: a review of neurophysiological and neuroimaging research. Alcohol Alcohol. 2014;49(2):198-206.

111. Riedel G, Platt B, Micheau J. Glutamate receptor function in learning and memory. Behav Brain Res. 2003;140(1-2):1-47.

112. Ward RJ, Lallemand F, de Witte P. Biochemical and neurotransmitter changes implicated in alcohol-induced brain damage in chronic or 'binge drinking' alcohol abuse. Alcohol Alcohol. 2009;44(2):128-35.

113. Guerri C, Pascual M. Mechanisms involved in the neurotoxic, cognitive, and neurobehavioral effects of alcohol consumption during adolescence. Alcohol. 2010;44(1):15-26.

114. Vetreno RP, Qin L, Crews FT. Increased receptor for advanced glycation end product expression in the human alcoholic prefrontal cortex is linked to adolescent drinking. Neurobiol Dis. 2013;59:52-62.

115. Philpot R, Kirstein C. Developmental differences in the accumbal dopaminergic response to repeated ethanol exposure. Ann N Y Acad Sci. 2004;1021:422-6.

116. LeMarquand D, Pihl RO, Benkelfat C. Serotonin and alcohol intake, abuse, and dependence: findings of animal studies. Biol Psychiatry. 1994;36(6):395-421.

117. Edenberg $\mathrm{HJ}$, et al. Variations in GABRA2, encoding the alpha 2 subunit of the GABA(A) receptor, are associated with alcohol dependence and with brain oscillations. Am J Hum Genet. 2004;74(4):705-14.

118. Kervern $M$, et al. Aberrant NMDA-dependent LTD after perinatal ethanol exposure in young adult rat hippocampus. Hippocampus. 2015;25(8):912-23.

119. Jacobus J, Tapert SF. Neurotoxic effects of alcohol in adolescence. Annu Rev Clin Psychol. 2013;9:703-21.

120. Bava S, et al. Longitudinal changes in white matter integrity among adolescent substance users. Alcohol Clin Exp Res. 2013;37(Suppl 1):E181-9.

121. Giedd JN. Structural magnetic resonance imaging of the adolescent brain. Ann N Y Acad Sci. 2004;1021:77-85.

122. Giedd JN. The teen brain: insights from neuroimaging. J Adolesc Health. 2008;42(4):335-43.

123. Guerri C, Bazinet A, Riley EP. Foetal Alcohol Spectrum Disorders and alterations in brain and behaviour. Alcohol Alcohol. 2009;44(2):108-14

124. Alfonso-Loeches S, Pascual M, Guerri C. Gender differences in alcoholinduced neurotoxicity and brain damage. Toxicology. 2013;311(1-2):27-34

125. Centonze $D$, et al. The link between inflammation, synaptic transmission and neurodegeneration in multiple sclerosis. Cell Death Differ. 2010;17(7):1083-91.

126. Amor $\mathrm{S}$, et al. Inflammation in neurodegenerative diseases-an update. Immunology. 2014;142(2):151-66.

127. Nisticò R, et al. Targeting synaptic dysfunction in Alzheimer's disease therapy. Mol Neurobiol. 2012;46(3):572-87.

128. Pignatelli $M$, et al. Synaptic plasticity as a therapeutic target in the treatment of autism-related single-gene disorders. Curr Pharm Des. 2013;19(36):6480-90.

129. Nisticò R, et al. Synaptic plasticity in multiple sclerosis and in experimental autoimmune encephalomyelitis. Philos Trans R Soc Lond Ser B Biol Sci. 2013;369(1633):20130162

130. McFarland HF, Martin R. Multiple sclerosis: a complicated picture of autoimmunity. Nat Immunol. 2007:8(9):913-9.

131. Mori F, et al. Interleukin-1 beta Promotes Long-Term Potentiation in Patients with Multiple Sclerosis. NeuroMolecular Med. 2014;16(1):38-51.

132. Kim Do Y, et al. Inflammation-mediated memory dysfunction and effects of a ketogenic diet in a murine model of multiple sclerosis. PLoS ONE. 2012;7(5):-e35476.
133. Sasaki A, et al. Microglial activation in early stages of amyloid beta protein deposition. Acta Neuropathol. 1997;94(4):316-22.

134. Tan ZS, et al. Inflammatory markers and the risk of Alzheimer disease: the Framingham Study. Neurology. 2007;68(22):1902-8.

135. Wang $\mathrm{Q}$, et al. beta-amyloid inhibition of long-term potentiation is mediated via tumor necrosis factor. Eur J Neurosci. 2005;22(11):2827-32.

136. Schmid AW, Lynch MA, Herron CE. The effects of IL-1 receptor antagonist on beta amyloid mediated depression of LTP in the rat CA1 in vivo. Hippocampus. 2009;19(7):670-6.

137. Kotilinek LA, et al. Cyclooxygenase-2 inhibition improves amyloid-beta-mediated suppression of memory and synaptic plasticity. Brain. 2008;131(Pt 3):651-64.

138. Jones RS, Lynch MA. How dependent is synaptic plasticity on microglial phenotype? Neuropharmacology. 2015;96(Pt A):3-10.

139. Hong $\mathrm{S}$, et al. Complement and microglia mediate early synapse loss in Alzheimer mouse models. Science. 2016:352(6286):712-6.

140. Diogenes MJ, et al. Extracellular alpha-synuclein oligomers modulate synaptic transmission and impair LTP via NMDA-receptor activation. J Neurosci. 2012;32(34):11750-62

141. Hirsch EC, Hunot S. Neuroinflammation in Parkinson's disease: a target for neuroprotection? Lancet Neurol. 2009;8(4):382-97.

142. Hunot S, Hirsch EC. Neuroinflammatory processes in Parkinson's disease. Ann Neurol. 2003;53 Suppl 3:S49-58. discussion S58-60

143. Xue $X$, et al. Baicalein ameliorated the upregulation of striatal glutamatergic transmission in the mice model of Parkinson's disease. Brain Res Bull. 2014;103:54-9.

144. Di Filippo M, et al. Neuroinflammation and synaptic plasticity: theoretical basis for a novel, immune-centred, therapeutic approach to neurological disorders. Trends Pharmacol Sci. 2008;29(8):402-12.

\section{Submit your next manuscript to BioMed Central and we will help you at every step:}

- We accept pre-submission inquiries

- Our selector tool helps you to find the most relevant journal

- We provide round the clock customer support

- Convenient online submission

- Thorough peer review

- Inclusion in PubMed and all major indexing services

- Maximum visibility for your research

Submit your manuscript at www.biomedcentral.com/submit
Biomed Central 